\title{
Surgery is the vessel of your soul
}

\author{
Martin Riegler
}

Accepted: 7 January 2021 / Published online: 18 January 2021

(C) Springer-Verlag GmbH, AT part of Springer Nature 2021

\section{Dear reader,}

welcome to the first 2021-issue of European Surgery including a spectrum of contributions offering particular themes, topics and motives related to our life. Surgery counts, because it cuts and aims to heal. The range of colours within this issue of European Surgery shines with stimulating, circulating, breathing, digestive and spherical scopes towards the essence of YOUR being: the sole actual perception of your momental atmosphere, mood and emotions. This moment equals the black holes of physics. Why? No information is allowed to get out of this quantum sized component of our existence. How? All informations are allowed to get in but nothing is allowed to get out, and this is why YOU do not remember birth and death! Got the argument?

If not, let us try again: slow motion step by step, one single note after the other continues to appear in the form of a motive. The motive is YOUR fugue and Bach rivers the fugue to provide the most important information and here comes the song and it goes like this: as you sit and read you might ask yourself: what the hell may the content of those lines be connected to my life, my family, my children, my grand children, my surgery, my scientific production, my paper writing, my list of arguments that I develop to gain power over my family, my people, my colleagues and my patients and my possessions! What the hell does that mean? Why is it important? Why does the author of those lines repeatedly state again and again that it is all about you, me, and us? Black holes hide information. Your moment contains all you know. You reason-

Doz. Dr. M. Riegler $(\bowtie)$

Reflux \& Health Care, Mariannengasse 10/4/9, 1090 Wien, Austria

martin.riegler@refluxordination.at ing does not allow to translate it all. Reality equals the part of it. How much you may perceive seems to be triggered by your state of emotions, temper and atmosphere. Art shows that, surgery, too. This is why you are not allowed to think thinking, to reason reasoning, to translate the entire moment of now. Here we reach the limits of and for our perception. Perception only allows the translation of it all into little parts and coins and ideas and segments and statements and thoughts and colours and sounds and kisses of atmospheres. Our perceptions only allow to abstract the momental stage of mood, temper and atmosphere. Remembrance creates the stream of emotion which in turn calls physics to read it and teach it in the forms of space time quanta future and past, size and extension, heat and freeze. Drops of blood count your surgeries.

Here we go: the smallest portions (units) of space time quanta are hard to translate. At some point the energy required to translate information within these small tiny quanta into our perception exceeds the energy kept within these space time quanta. As a consequence, energy within these quanta increases and resolution ceases to work, all information is kept within the space time quanta black holes and we are not allowed to know and see and assess what may be going on there. We are kept away from information. Here mythology comes into play. Surgery and mythology share an identical methodological approach: mythology and surgery (medicine) aim to translate images into treatable signs. As such YOU take the patient history, evolve the large spectrum of diagnostic tests to collect data for a tailored cause-related therapy. Applause! Are you always allowed to do it this way? Are you always kept away from superficial conduct of reasoning? Who are you? Are you monitored, ruled, controlled by a global web based technology following the interests of those who program and format this fascinating new god? Google orders days (GOD!). Where 
did the dog run? Whom do you serve? Stan and Oliver? Lakes of thunder? Global warming interests? Poisoned prisoners of a new wave of interaction, where a small tiny sphere interacts with the inventions of man, where a small sized natural phenomenon interacts with the beautiful achievements of man? Where did your towns go? Where did your money go? Where did your hopes and believes go? Where did your computers go? Where did you go? Going home, ten years after? Us and them? Pink and Floyd? Beatles did not suffer from locks down. Rockers did not run out of material. Rasta did not get out of slavery, but they knew what they have been talking about: it is all there out there, just open your eyes and look within. Our world turns round, vaccinates and offers the assessment of our roots. Unfortunately you are not ready yet to get the message. It seems we have to await the episode that has to follow after the lock-down of reasoning. Not the streets are empty, not the restaurants are empty, not the schools are empty, while the hospitals get full and fuller, rich and richer, not the airports are empty, not the free ways and high ways are empty, not the shops are empty. But what got empty? The answer: your brains, your hearts, your thoughts, your phantasy, your dreams and your emotions. While the climate warmed up, you became cold, lonely and anxious. Anxiety fosters believe. In that you trust. As such you tailor the treatment of your current condition. You have been weak, as a consequence you became soft and this in turn shows up as a global warning: may we have over done? And again YOU are to be congratulated that you made it to come up to this point of the editorial, that you made it to this sentence and those words, your passion shows that you may be interested. Why? Because our current possibility offers time to rethink our way of life, to reformat our hard disk storage board. When you read those lines your remembrance proofs, that days are getting longer. There is more time offering positive sun shine. As such we realise to be part of a magic mechanism. At this point we unite.

Your surgery happens under the shelter of the universe, may it be round, spheric or flat. Your treatments are fascinating orchestrations of diagnosis and therapy. Your actual stage of mood may be happy, nervous or disparate. Your momental groove may be positive or negative or both. Your wave of emotions may split splash over the desires of your colleagues. You are treating tuff gong cases. Your actions aim to elucidate the applause by others. Who applauses and admires the boss? Who is the peak of the mountain? Geology teaches that the peaks of our beloved mountains came from Africa, Australia, followed by the rocks composed of highly compressed sea soil calcium shores. Beyond that geological macro-phagocytosis kept us free for having oil, gas and metals. At best you may get $100,000,000$ dollars per $24 \mathrm{~h}$ of a single day, from Monday to Sunday, seven days a week, and you might have been working like a dog. Resources count, matter and form, this is why many nations lacking those resources justify themselves by driving philosophical ideas: dead at arrival but promising you a lot.

Without oil and gas there would not be enough wood to heat our alternative housings. As a consequence, we would have to go back to Africa, to see our brothers and sisters down there. Why? We are all brothers and sisters, rooting from a single family, that has been originating from Africa, many million years ago. Theoretically we also could have rooted from the Americas, but so far we lack respective evidence supporting this theory. As a matter of fact, days get longer and this fact correlates with a greater yield for assessing sunset and sunrise before and after your surgeries. Do not overdo and do not try to become a hero. Before the ideas of heroes have been invented our ancestors mingled into the skies. They did it with passion for orientation and positive atmosphere. As such they found out. Here we go. Within the next weeks YOU will cut, dissect, suture and resect towards YOUR spring equinox.

During a full year we may be fascinated by the $360^{\circ}$ full circle of the zodiacs dancing around the night time skies. This dance of the "gods" relates to the wobble of the earth axis which develops due to the spacetime interactions between the sun, the earth and our neighbouring planets and our moon. We are part of the milky way, even your surgeries happen within the milky way! Even the ions and the water collected (logos) within your body and the bodies of those you are allowed to operate (written consent!!) are the same, when compared to those "out there" in the remote galaxies of our Hubble telescope designed universe (is there an other one, too?).

The wobble of the earth axis reads out the large years, i.e. approximately 26,000 solar years. During the large years the tilt of the earth axis dislocates at 1 degree every 72 years, this is 25,920 years for $360^{\circ}$ full circle of the sky $(=12$ large months; each month falls within the sign of a particular zodiac, i.e. waterman, fish, Aries, Taurus/bull etc.) (Fig. 1). Therefore, over many thousand years, at spring equinox, the sun seems to rise against the background of different signs of the twelve zodiacs (12 hours, tribes, zodiacs, columns of a temple, windows of palace or church, and so forth; numbers of interest: so called precision numbers: 12,$24 ; 36,72$ etc.). The sun stays within a particular zodiac sign for approximately 2160 years. As such the particular zodiac sign houses the sun. When this happens repeatedly for many years, you may read, that the hero fell within the lions (ref. Gilgamesh epos) or that the sun god fought the Taurus/ bull (ref. Gilgamesh; Ancient Egyptian Pyramid texts, coffin texts). In our times, and you may check it out within the next weeks of YOUR LIFE (21.3.2021), the sun, YOUR SUN, seems to rise against the zodiac sign of the fish. At spring equinox, the sun is doing this since approximately 2020 years. Before that, at spring 


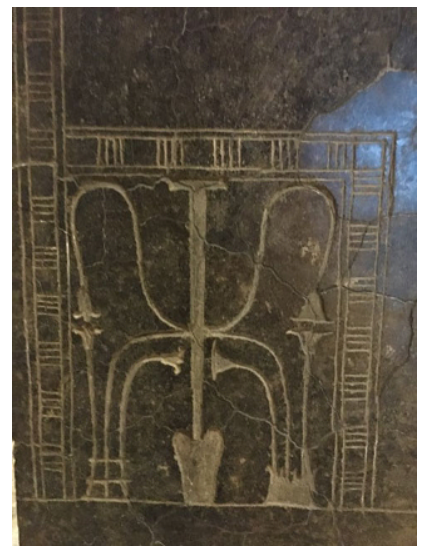

Fig. 1 The image cartoons ancient comic knowledge regarding the dynamic, wave - like, periodical wobble of the tilt of the earth axis (precession) which is responsible for the sequence of zodiacs housing the sun during spring equinox, as described in the text. Image obtained at the Luxor temple, Egypt

equinox, the sun rose against the zodiac sign of Aries (ram), before that it had been the Taurus/bull and so forth. This fact explains why the respective animals have been so important during cultures 2000, 3000, 4000 years ago and so forth. Within the next 300 years the spring equinox sun will be housed by the zodiac sign of the waterman. This fact relates to the song about Aquarius within the famous hippie musical "Hair". Let us turn to the autumn equinox.

During autumn equinox 2021 sun will rise against the zodiac sign of the virgin. May it be that the fish, Jesus and virgin story has been created to remember these cosmic constellations of the zodiacs? As such the spring/autumn equinox zodiac pairs of Aries/Libra, Taurus-Bull/Scorpion, Gemini/ Sagittarius, Cancer/Capricorn, Lion/Waterman and so forth have been incorporated into the iconography of cultures where the respective zodiacs have been "housing" the sun during the spring and autumn equinox, respectively.

Think of the surgeons in the old, middle and new ancient Egyptian kingdom, the cultures arising in Mesopotamia or the Levante, those of North and South America? This may explain why sculptural representations of Lion and Capricorn cover the columns within the ruins of Gobekli Tepe, Turkey (around 10,000 years before present), why the bull and the scorpion became so important for cultures designed 3000-4000 years BC (Indus valley, Crete, old Egyptian Kingdom) and why the new Egyptian kingdom and other cultures around that time (2000 BC-0) favoured the symbols of Aries and Libra (ancient Assyrian, Persian, Greek, Levantine culture and so forth) (Fig. 2). May it be that man always intended to mirror the heavens here on earth (Fig. 3)? May it be that our ancestors oriented their architecture, their monuments, palaces, streets, cities, temples, churches and grave

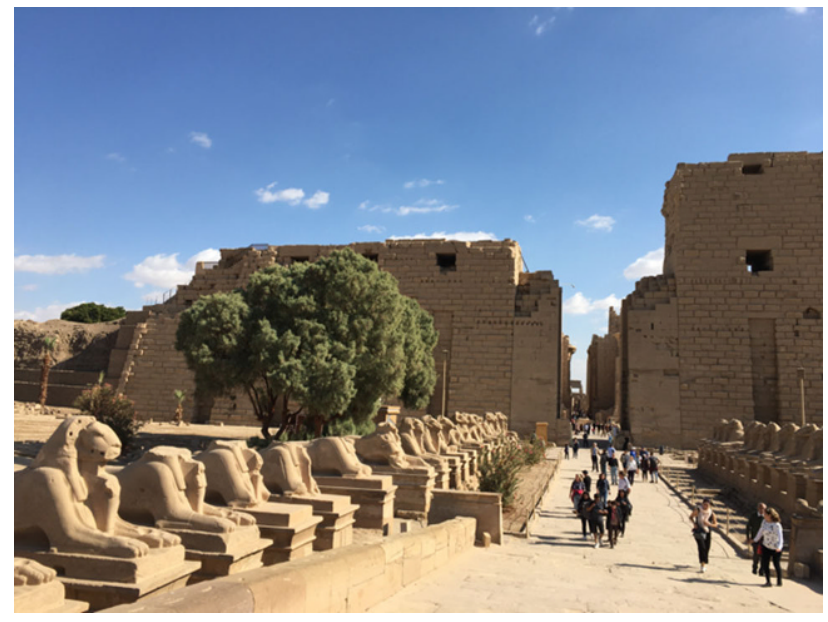

Fig. 2 This image shows the remnants of the ram alley (Karnak), that has been created during the period when the zodiac sign Aries (ram) housed the sun during the spring equinox. The image cartoons the idea that architecture mirrors the heavens, as described in the text. Image obtained in Karnak, Egypt

yards according to the heavens and skies to mirror the images from above (Figs. 3 and 4)? In addition to reflecting the human conditions here on earth, may it not have been the fascination for the night skies and zodiac constellations, which motivated our ancestors to build pyramids, temples and churches to foster our attention towards that part of the universe, which homes our solar system and neighbouring stars, planets and comets (Fig. 3)? May it be that it all aimed to mirror the heavens and to show us that we are in fact what we are: part of the galaxy that mammals used to name the milky way (Figs. 3 and 4)? How would the dinos have thought about the moon and the sun and the zodiacs and the galaxy? Would they have named

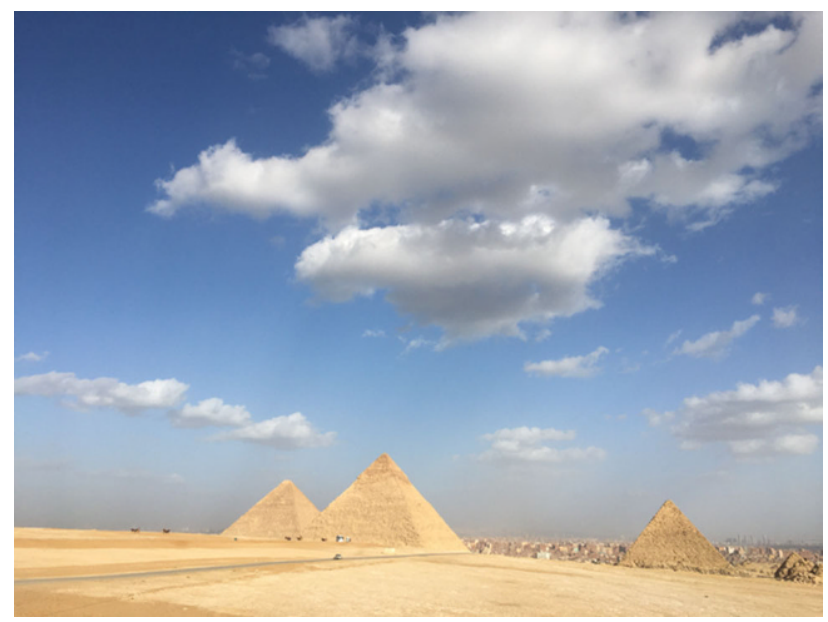

Fig. 3 This image shows the great pyramids of Giza, Egypt. The orientation of the pyramids has been demonstrated to mirror the constellation of the Orion belt stars. Thus the image cartoons the idea of the author, that ancient architecture mirrors the heavens, as described in the text. Image obtained in Giza, Egypt 


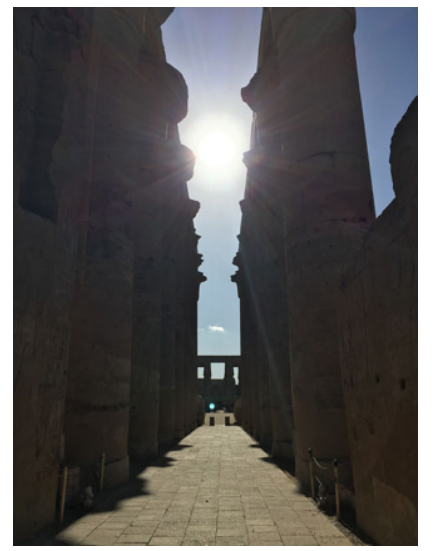

Fig. 4 This image shows sunrise at winter solstice at the great temple of Karnak, Egypt. Note that the axis of the temple of Karnak orients along the axis of the position of the sun during sunrise at winter solstice (21.12). The image cartoons the idea of the author, that ancient architecture mirrors the heavens, as described in the text. Image obtained at winter solstice 2020

it their "shell of the egg"? We could ask those, who share the ancestry of the dinos: the birds! Beep it out now: "Good vibrations for the nations".

As such we fly away within the beautiful and meaningful cosmic vibrations. Let us thank our planet for taking us around the sun and through the twisted turns of the milky way. Let us thank our planet for its passion to take us as we are: good and evil, exciting and destroying, full and empty of motivation and death creating sprit. Once upon a time the last words of a cancer patient would have been: "The universe is the vessel of my soul". As such we diagnose: all is one. The therapy: enjoy now, the black holes in your sky, where all remembrance comes after, beyond and before, but not now.

Stay tuned, foster you, be you,

sincerely yours

Martin Riegler.

Acknowledgements the author thanks his patients from whom he learns so much about the beauty and fascination of life and its manifestations. In addition this is to thank all who contribute to support the Europan Surgery project. Finally the author thanks Jan Assmann, Karl Corino, Graham Hancock, Robert Bauval, Robert M. Schoch, Heinrich Kusch and Israel Finkelstein for their important and highly valuable contributions, which motivated the research, as outlined in the text.

Conflict of interest M. Riegler declares that he has no competing interests.

Publisher's Note Springer Nature remains neutral with regard to jurisdictional claims in published maps and institutional affiliations. 